\title{
A Potential Biomarker for Acute Kidney Injury in Preterm Infants from Metabolic Profiling
}

\author{
Lindsey E. Romick-Rosendale ${ }^{1}$, Kurt R. Schibler ${ }^{2}$, and Michael A. Kennedy ${ }^{1 *}$
}

${ }^{1}$ Department of Chemistry and Biochemistry, Miami University, Oxford, $\mathrm{OH} 45056$, USA

${ }^{2}$ Perinatal Institute, Cincinnati Children's Hospital Medical Center, Cincinnati, OH 45229, USA

\begin{abstract}
Background: Currently used biomarkers for acute kidney injury (AKI), namely $\mathrm{Ngal}, \mathrm{SCr}$, and BUN, are inadequate for timely detection of $\mathrm{AKI}$ in preterm infants.

Methods: Nuclear magnetic resonance (NMR) spectroscopy-based metabolic profiling was conducted on urines from 20 preterm infants to determine if novel metabolic biomarkers could be identified for early detection of AKI. Urines were collected from every patient each day for the first 14 days of life. NMR spectra were measured for all urines and metabolic profiling analysis conducted.

Results: One metabolite, carnitine, increased significantly in urines of three extremely low birth weight (ELBW) infants starting on day five of life. The three affected infants either received prolonged antibiotic treatment, extended treatment with indomethacin, or both. One ELBW patient who received both treatments and reached the highest urinary carnitine level died on day 10 of life due to localized gut perforation complicated by suspected AKI.

Conclusions: It was concluded that carnitine increased in the three neonates in part due to antibiotic- and/ or indomethacin-induced AKI. It is hypothesized that combined antibiotic and indomethacin treatment promoted AKI resulting in reduced proximal renal tubule reabsorption of carnitine and that $\beta$-lactam antibiotics blocked renal carnitine uptake by human organic cation transporter, hOCTN2.
\end{abstract}

Keywords: Acute kidney injury; Preterm infant; Necrotizing enterocolitis; NMR; Metabonomics; Neutrophil gelatinase-associated lipocalin (Ngal); Carnitine; Blood Urea Nitrogen (BUN)

\section{Introduction}

The survival rates of preterm infants, particularly those in the very low birth weight (VLBW) population born weighing less than $1500 \mathrm{~g}$, has continued to increase dramatically in recent years due to improved neonatal care, advances in medical instrumentation and administration of more effective medications. However, similar advances in the treatment and diagnosis of acute kidney injury (AKI) in premature infants have yet to be seen. In a recent neonatal study, the incidence of AKI in hospitalized newborns ranged from 8 to 24\% [1]. The study found that factors such as VLBW, patent ductus arteriosus accompanied by administration of antibiotics and nonsteroidal anti-inflammatory drugs, and a low Apgar score, were all associated with progression of AKI [1]. Neonates and infants, aged one day to one year, accounted for $57 \%$ of the total non-survivor group of individuals afflicted with AKI [2]. An increase in the survival rates of preterm infants has also resulted in an increase in the incidence of AKI in this patient population. AKI, previously called acute renal failure, is clinically recognized by an abrupt reduction in renal function or urine output [2]. The term AKI was chosen to reflect the full spectrum of acute kidney failure, reflecting that a sharp decline in kidney function is often a result of injury that causes functional or structural modifications in the kidneys [3].

The most common form of AKI in hospitalized patients is acute tubular necrosis (ATN) [4], which is caused by toxic or ischemic injury to the tubular epithelial cells resulting in tubular dysfunction [5]. Currently, clinicians diagnose AKI based on changes in serum creatinine (SCr) concentration [6] and blood urea nitrogen (BUN) levels [3]; however, changes in both $\mathrm{SCr}$ and BUN levels are late consequences of kidney damage [7] and are not direct markers injury itself [8]. Lavery et al. also pointed out that SCr concentrations fluctuate significantly over the first several weeks of a newborn's life, with initial urine levels reflecting those of maternal kidney function due to placental transfer [9], further complicating the use of $\mathrm{SCr}$ as a potential marker of AKI in preterm infants. Renal tubule damage, as seen in cases of ATN, can be insufficient to cause a change in currently monitored parameters of kidney function such as SCr or BUN levels [10]. Failure of early diagnosis and treatment of AKI is partially due to lack of sensitive and specific renal markers to allow early detection of impending AKI [11]. Ideal biomarkers of AKI would be up-regulated soon after renal injury and would be independent of glomular filtration rate (GFR). In premature infants, extra-uterine renal development impacts the ability to accurately monitor changes in potential AKI biomarkers, which take place over the first few days or weeks of life [12], creating a further challenge in the discovery of biomarkers of AKI in this population.

The antecedents of AKI are often caused by various factors, with nephrotoxic and ischemic insults being common [4]. Many antibiotics, particularly aminoglycosides, are known to cause tubular injury in term and preterm neonates [13]. For example, gentamicin, which is commonly used to treat early bacterial infections in neonates, affects glomerular and tubular function and is a known risk factor for the development of AKI [14]. Indomethacin, which is used to treat patent

*Corresponding author: Michael A. Kennedy, Department of Chemistry and Biochemistry, Miami University, Oxford, Ohio 45056, USA, Tel: 513-529-8267; Fax: 513-529-5715; E-mail: kennedm4@muohio.edu

Received December 09, 2011; Accepted January 28, 2012; Published February 02, 2012

Citation: Romick-Rosendale LE, Schibler KR, Kennedy MA (2012) A Potential Biomarker for Acute Kidney Injury in Preterm Infants from Metabolic Profiling. J Mol Biomark Diagn S3:001. doi:10.4172/2155-9929.S3-001

Copyright: (c) 2012 Romick-Rosendale LE, et al. This is an open-access article distributed under the terms of the Creative Commons Attribution License, which permits unrestricted use, distribution, and reproduction in any medium, provided the original author and source are credited 
Citation: Romick-Rosendale LE, Schibler KR, Kennedy MA (2012) A Potential Biomarker for Acute Kidney Injury in Preterm Infants from Metabolic Profiling. J Mol Biomark Diagn S3:001. doi:10.4172/2155-9929.S3-001

ductus arteriosus, is also known to cause renal failure [15]. Furthermore, studies have also indicated that sepsis, which can be associated with localized gut perforation, continues to be a significant factor in causing AKI [4]. The various factors influencing the onset of AKI trigger the disorder via different mechanisms; however, a common pathway of ATN en route to AKI has been identified [16].

Neutrophil gelatinase-associated lipocalin (Ngal), a protein which binds siderophores (iron-chelating molecules secreted by microorganisms) [17], has previously been identified as a valuable biomarker of AKI in adult patients [18]; however, it has poorer prognostic value for AKI in children [11]. Ngal binds siderophores and inhibits growth of bacterial strains that rely on siderophores as a source of iron [19]. In adults, increased urinary and serum Ngal levels during AKI is a result of NGAL expression rapidly induced in the nephron in response to renal epithelial injury [20]. Lavery et al. found that Ngal alone is not an effective marker of AKI in VLBW infants due to overall variability in Ngal concentrations among preterm infants in comparison to other populations [9]; however, it was found that infants who did not have other clinical indicators for AKI also tested negative for AKI based on Ngal screening [9]. It was concluded that additional studies were needed to determine whether Ngal was a more specific biomarker of AKI than SCr in the VLBW class of newborns.

In the study reported here, we have further analyzed the urine samples from the study conducted by Lavery et al. [9] with the goal of identifying a metabolic biomarker of AKI that is more reliable than Ngal or SCr. The technique of nuclear magnetic resonance spectroscopy (NMR)-based metabonomics was used for metabolic profiling. NMRbased metabonomics is a powerful method for characterizing metabolic profiles of biological fluids [21,22] including urine, feces, serum, and spinal fluid and can offer information concerning the metabolic processes of organs such as the liver, kidneys, brain and stomach [23, 24]. High-field $(850 \mathrm{MHz})$ nuclear magnetic resonance spectroscopy (NMR)-based metabolic profiling was used to characterize urines of 17 VLBW and three extremely low birth weight (ELBW) preterm infants collected over the first 14 days of life. Significant elevation in the concentration of one metabolite, carnitine, was observed in three extremely low birth weight (ELBW) infants weighing less than $1000 \mathrm{~g}$. Day-to-day changes in carnitine levels of these three patients appeared much more stable compared to the corresponding changes in Ngal levels, which fluctuated dramatically in the same patients. Elevated carnitine concentrations in the three ELBW patients could be directly attributed to a dysfunction in the renal tubular epithelia, which is associated with AKI, as will be discussed in detail.

\section{Methods}

\section{Study population}

Samples were obtained from a study conducted at Cincinnati Children's Hospital Medical Center (CCHMC) in which 20 premature infants with birth weights (BWs) between 500 and $1500 \mathrm{~g}$ were enrolled at three neonatal intensive care units in Cincinnati, Ohio (University Hospital, Good Samaritan Hospital, and Cincinnati Children's Hospital). The Institutional Review Board at each of the three hospitals reviewed and approved the study and the informed consent documents. Infants were included if their BW was between 500 and $1500 \mathrm{~g}$ and parental consent was granted. Patients were differentiated into the following four BW categories 500-750, 751-1000, 1001-1250, and 1251-1500 g. Infants were excluded if a significant congenital anomaly was identified. One patient in the 500-750 BW category died on day 10. All preterm infants were on a standard continuous $20 \mathrm{mg} /$ $\mathrm{kg}$ /day dosage of carnitine, except that two of the three ELBW infants received elevated dosages of $30 \mathrm{mg} / \mathrm{kg} / \mathrm{day}$ and $35 \mathrm{mg} / \mathrm{kg} /$ day, however the third ELBW infant, who died on DOL 10, received the standard 20 $\mathrm{mg} / \mathrm{kg} /$ day dosage.

\section{Sample collection}

Urine was collected from diapers using a cotton ball, or bladder catheter if present, within the first $24 \mathrm{~h}$ of life and daily until day of life (DOL)-14. The infant's diapers were checked every $4 \mathrm{~h}$ and collected urine was transferred to a refrigerator located at each study site. Urine samples were relocated to a $-80^{\circ}$ freezer within $48 \mathrm{~h}$ of collection.

\section{Preparation of biological fluids for NMR analysis}

Urine samples were stored at $-80^{\circ} \mathrm{C}$ after collection until prepared for NMR measurements. Samples were thawed on ice prior to preparation for NMR analysis. A one-milliliter aliquot of each sample was centrifuged for 10 minutes at $2655 \mathrm{xg}$, and then 350 microliters of clear urine was pipetted into a 1.5 milliliter microcentrifuge tube. A volume of 350 microliters of buffer $\left(300 \mathrm{mM} \mathrm{KH}_{2} \mathrm{PO}_{4}, 2 \mathrm{mM} \mathrm{NaN}\right.$ $0.2 \%$ trimethylsilyl propionate (TSP) in $20 \% \mathrm{D}_{2} \mathrm{O}, \mathrm{pH} 7.4$ ) was added

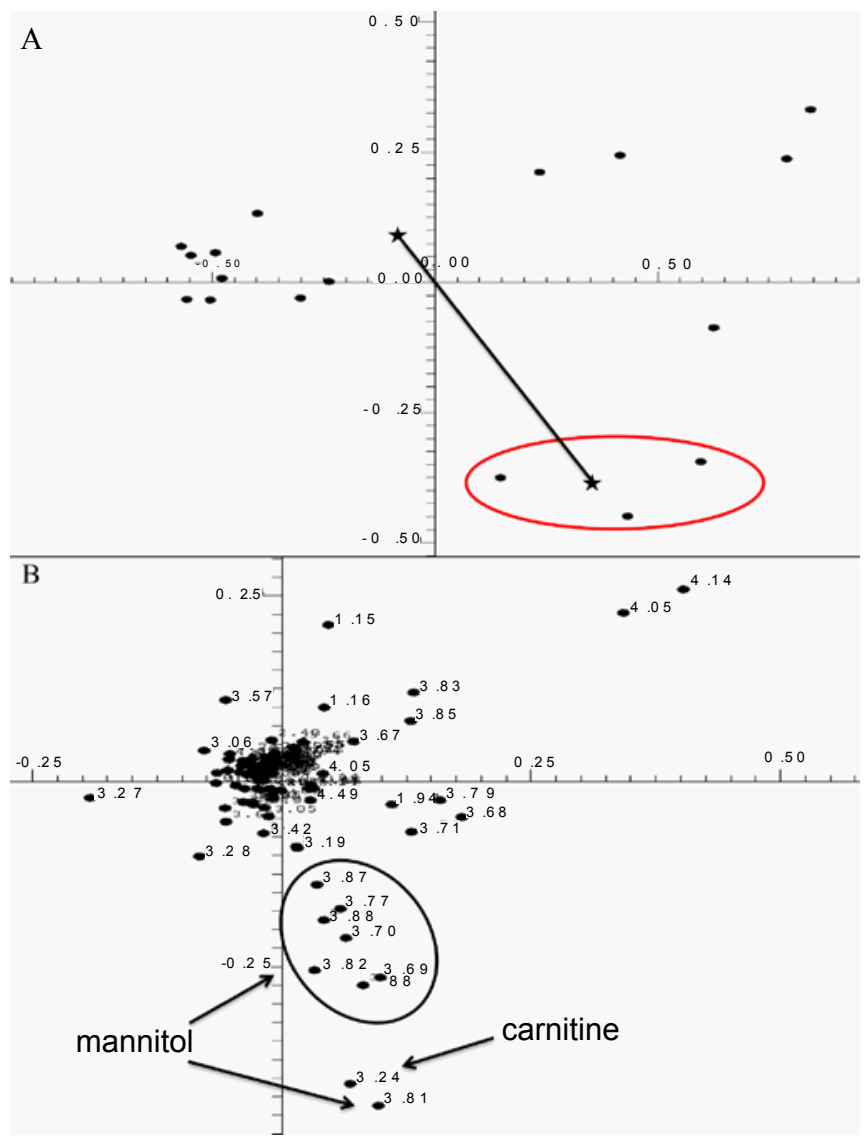

Figure 1: A) Two-dimensional scores plot of human urine samples of DOL 8 calculated using the first two principal components of the PCA. The cluster circled at the lower right represents urine samples of the three ELBW infants suffering from suspected renal injury. The stars indicate the centroids of the two clusters discussed in the text, and the line joining the centroids represents the Mahalanobis distance discussed in the text. B) The loadings plot corresponding to the scores plot in Figure 1A. The solid ovals encircle groups of resonance frequency buckets that belong to the same metabolite and are labeled according to the identity of the metabolite. 
to each urine sample. A volume of 600 microliters of each urine/buffer mixture was then pipetted into a $5 \mathrm{~mm}$ NMR tube (Norell ST500-7).

\section{NMR data collection and processing}

All NMR experiments were carried out on a Bruker Avance ${ }^{\mathrm{Tt}}$ III spectrometer operating at $850.10 \mathrm{MHz}{ }^{1} \mathrm{H}$ frequency and equipped with a room temperature $5 \mathrm{~mm}$ triple resonance probe with inverse detection and controlled by TopSpin 2.1.4 (Bruker, Germany). All experiments were conducted at $298 \mathrm{~K}$. All data was collected using a spectral width of $20.0 \mathrm{ppm}$. Three ${ }^{1} \mathrm{HNMR}$ experiments, optimized by Bruker (Bruker BioSpin, Billerica, MA) for use with metabonomic studies were run on all samples: a standard 1D presaturation (zgpr), the first increment of a 1D NOESY (noesygppr1d), and a CPMG (cpmgpr1d) experiment. All experiments included presaturation of the water peak. The transmitter offset frequency (O1) was set to $4002.80 \mathrm{~Hz}$ to obtain optimal water suppression. The $90^{\circ}$ pulse width was determined for every sample using the automatic pulse calculation feature in TopSpin. All pulse widths were between 13 and $16 \mu$ s. Water suppression was achieved by irradiation of the water peak during a recycle delay of 4.0 s with a pulse power level of $55.92 \mathrm{~dB}$.

The zgpr experiment was used to screen samples and to assure that presaturation and shimming was sufficient for reliable data collection. To check the shimming, the full width at half height (FWHH) of the TSP peak was measured. The shimming was deemed acceptable when the FWHH was less than $0.9 \mathrm{~Hz}$. The one-dimensional zgpr [1] HNMR spectra were acquired using 2 transients and 2 dummy scans, $65 \mathrm{~K}$ points per spectrum giving an acquisition time of $1.92 \mathrm{~s}, 0.03 \mathrm{~Hz}$ of exponential line broadening, and a recycle delay of $4 \mathrm{~s}$. Once the spectrum was determined to be of acceptable quality the other two experiments were run. The first increment of the 1D NOESY experiment was collected using 16 transients with 4 dummy scans, $65 \mathrm{~K}$ points per spectrum giving an acquisition time of $1.92 \mathrm{~s}$, a mixing time of $10 \mathrm{~ms}$, and apodized using a gaussian line broadening parameter of 0.01 , and a $4 \mathrm{~s}$ recycle delay. The CPMG experiment was collected in order to filter broad peaks present in the spectrum. The CPMG experiment was collected using 128 transients with 4 dummy scans, $65 \mathrm{~K}$ points per spectrum giving an acquisition time of $1.87 \mathrm{~s}$, a $\mathrm{T}_{2}$ filter loop of 128 with an echo time of $1 \mathrm{~ms}$, apodized using $-.01 \mathrm{~Hz}$ of exponential line broadening, and a $4 \mathrm{~s}$ recycle delay. All NMR spectra were phased, baseline corrected, and corrected for chemical shift registration relative to TSP in TopSpin 2.1.1 (Bruker Biospin, Billerica, MA).

\section{Box and whisker plot analysis}

Box and Whisker plots were generated in excel using a template provided by vertex 42 .com.

\section{Principal component analyses (PCA)}

The data were subjected to multivariate statistical analysis using AMIX software version 3.9.5 (Bruker Biospin, Billerica, MA). NMR spectra were binned into $0.01 \mathrm{ppm}$-wide buckets over the region $\delta$ 10.0 to $0.2 \mathrm{ppm}$. The region of $\delta 4.75-5.1$ was excluded from analysis to avoid effects of imperfect water suppression. Buckets with variances $<5 \%$ were also excluded from PCA. Initially, unsupervised PCA was performed without consideration of class information. Rousseau et al. discusses the algorithm employed to calculate the principal components [25]. Visualization of the data was accomplished by inspection of the PCA scores and loadings plots.

\section{Mahalanobis distance and F-Value calculations}

Mahalanobis distance calculations were performed in MatLab using a method to quantitatively and statistically analyze cluster separations in PCA scores plots of NMR data developed by Goodpaster and Kennedy [26].

\section{Identification of metabolites}

Experimental NMR spectra were compared with those of candidate metabolites using the ChenomX NMR Suite (ChenomX Inc., Edmonton, Alberta, Canada). The ChenomX database was used to filter for resonance frequencies at chemical shifts corresponding to buckets identified as significant in the loadings plot. ChenomX database spectra for candidate metabolites were inspected to determine if peak patterns matched those observed in the experimental data. If the ChenomX database spectrum unambiguously matched a group of significant buckets identified in the loadings plot, direct assignment of the metabolite was made. In most cases, incorrect candidate metabolites could be eliminated by inspection and comparison with ChenomX database spectra due to mismatched peak patterns and/or intensities.

\section{Quantification of metabolites}

Urine Ngal levels were determined for the previous study as described by Lavery et al.[9]. Carnitine levels were determined using the AMIX software. Specifically, an internal chemical shift and concentration standard, TSP, was added to each urine sample prior to NMR data collection. Once carnitine was identified using the AMIX software, the concentration of carnitine was determined by matching the intensity of the carnitine peaks in the database spectrum with the intensity of the observed carnitine peaks in the experimental spectrum. Therefore, the absolute concentration of the carnitine peaks was calibrated relative to the internal TSP standard.

\section{Results}

\section{NMR metabonomics analysis}

PCA of urines indicated no patient differentiation from DOL $1-4$. Beginning on DOL 5 , three patients began to group separately from the rest of the population based primarily on increased urinary carnitine (Figure 1A), which was absent in urines of all other patients. The magnitude of cluster separation was evaluated by calculating the Mahalanobis distance $\left(D_{M}\right)$ between the cluster centroids and the statistical significance of cluster separation was evaluated by calculating an F-value (Figure $1 \mathrm{~A}$ ). The $\mathrm{D}_{\mathrm{M}}$ of 5.887 between carnitinepositive and carnitine-negative group centroids, and F-value of 38.394 (critical F-value 3.739), indicated statistically significant group separation. Separation of these patients based on elevated carnitine levels continued from DOL 5 until the end of the study on DOL 14 One carnitine-positive patient died on DOL 10. Visual inspection of all NMR spectra (Figure 2) confirmed that carnitine was only present in the three patients identified as outliers in Figure 1A.

The three carnitine-positive patients were all members of the ELBW (500-750g) class of preterm infants. Figures 3A through 3D show how carnitine and Ngal levels varied with respect to body weight for two carnitine-negative patients (Figure $3 \mathrm{~A}$ and $3 \mathrm{~B}$ ) and two carnitinepositive patients (Figure $3 \mathrm{C}$ and $3 \mathrm{D}$ ). Ngal levels of carnitine-negative patients fluctuated strongly during the 14-day study (Figure 3A and 3B) while their body weights increased steadily and there were no other indications of renal injury. Figure $3 \mathrm{C}$ shows data for a carnitinepositive patient. The Ngal and carnitine levels began to climb on DOL 4 and DOL 5, respectively. However, whereas Ngal peaked at about 400 $\mathrm{ng} / \mathrm{mL}$ on DOL 6 and then steadily decreased to $\sim 100 \mathrm{ng} / \mathrm{mL}$ on DOL 9 , the day before the patient's death on DOL 10, carnitine started from 


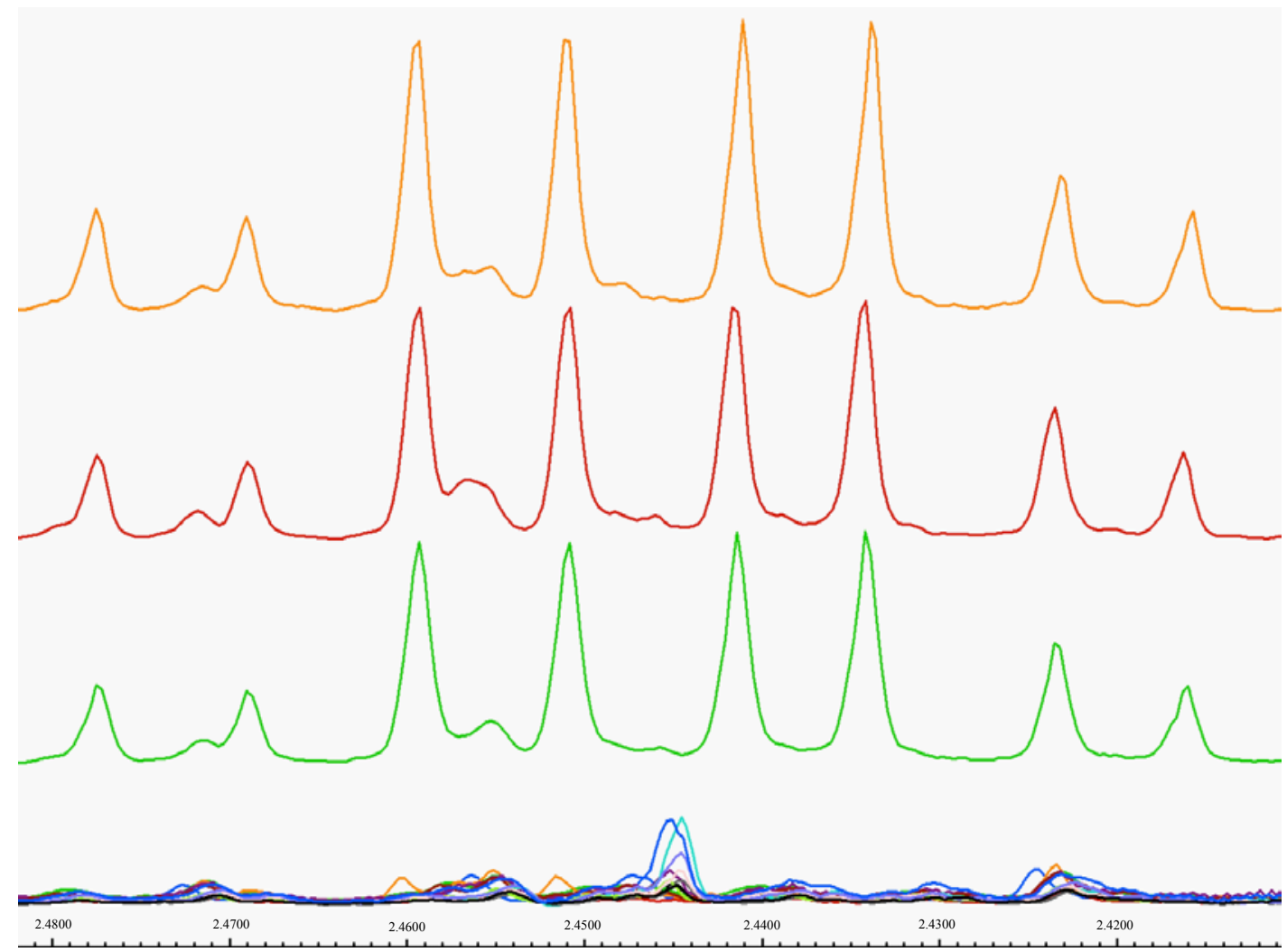

Figure 2: A) Urine NMR spectra in the region from 2.48-2.41 ppm containing a carnitine-specific multiplet for all patients on DOL 8 . NMR spectra for three patients displaying elevated carnitine levels are shown at the top.

$34.9 \mu \mathrm{g} / \mathrm{mL}$ on DOL 4 and steadily increased to $162 \mu \mathrm{g} / \mathrm{mL}$ on DOL 9 , the day before the patient's death on DOL 10. Based on Ngal values alone, one might conclude that this patient was moving away from indication of AKI, however carnitine steadily increased until death. This patient lost substantial body weight $(\sim 40 \%)$ just before Ngal and carnitine levels began to rise and the infant never regained significant body weight. Figure 3D shows data from another carnitine-positive patient. This patient displayed strong fluctuations in Ngal over the first week of life (ranging from $<200$ to $>800 \mathrm{ng} / \mathrm{mL}$ ) and then the Ngal levels dropped to values seen in other healthy patients. Interestingly, carnitine began to increase starting on DOL 5 reaching a maximum concentration of $151 \mu \mathrm{g} / \mathrm{mL}$ and fluctuated between $33.1 \mu \mathrm{g} / \mathrm{mL}$ and $106 \mu \mathrm{g} / \mathrm{mL}$ between DOL 5 and DOL 14. Over the same time period, the infant steadily gained weight resulting in a $20 \%$ increase in body weight.

Figure 4 illustrates how existing clinical markers of renal damage, Ngal, SCr, and BUN, varied in carnitine-positive and carnitine-negative patients from DOL 5-14. All three carnitine-positive patients presented with higher levels of all three existing renal function markers compared to carnitine-negative infants.

\section{Discussion}

Current biomarkers for detection of AKI in premature infants, Ngal, SCr, and BUN, are inadequate [27] limited by poor sensitivity and specificity [11]. AKI is usually diagnosed indirectly by a measured rise in SCr, which is thought to indicate a reduction in GFR [28]. However, $\mathrm{SCr}$ concentration does not accurately mirror GFR in the non-steady- state condition of patients with AKI since SCr levels are also influenced by the degree of distribution, the rate of production, and continued renal development in preterm infants and neonates $[29,30]$. Although Ngal has been identified as a useful biomarker for diagnosis of AKI in adults, the marker has not proven as useful in determining renal failure in children, and perhaps more critically, in preterm infants [9]. Ideally, biomarkers of AKI in preterm infants would present soon after renal injury and would be independent of GFR. Potential biomarkers of AKI might show up in urine during tubular inflammation or tubular dysfunction, leading to enzymes, other proteins, or small molecule metabolites in urine that are generally filtered at the glomerulus [31].

Although current prognostic indicators fall short in their ability to predict acute renal failure in its earliest stages [11, 28, 29], they are still considered the best existing measures of kidney function $[3,6,18]$. Therefore, we evaluated carnitine as a potential marker of early AKI by comparing its levels to currently used clinical biomarkers in carnitinepositive and carnitine-negative preterm infant patients. Carnitinepositive patients exhibited higher levels of Ngal, SCr, and BUN compared to the 17 carnitine-negative patients suggesting that these patients were experiencing greater renal dysfunction. Collectively, our data suggest that multiplexing a carnitine-positive measurement with other markers for AKI may lead to a more sensitive, specific, and accurate predictive capability of AKI in preterm infants.

Eighteen of twenty infants in the study, including the ELBW infant that died on DOL 10 , received daily continuous dosages of carnitine of $20 \mathrm{mg} / \mathrm{kg} /$ day, while the two other ELBW infants received $30 \mathrm{mg} /$ $\mathrm{kg} /$ day and $35 \mathrm{mg} / \mathrm{kg} /$ day; importantly, the ELBW who died on DOL 
Citation: Romick-Rosendale LE, Schibler KR, Kennedy MA (2012) A Potential Biomarker for Acute Kidney Injury in Preterm Infants from Metabolic Profiling. J Mol Biomark Diagn S3:001. doi:10.4172/2155-9929.S3-001

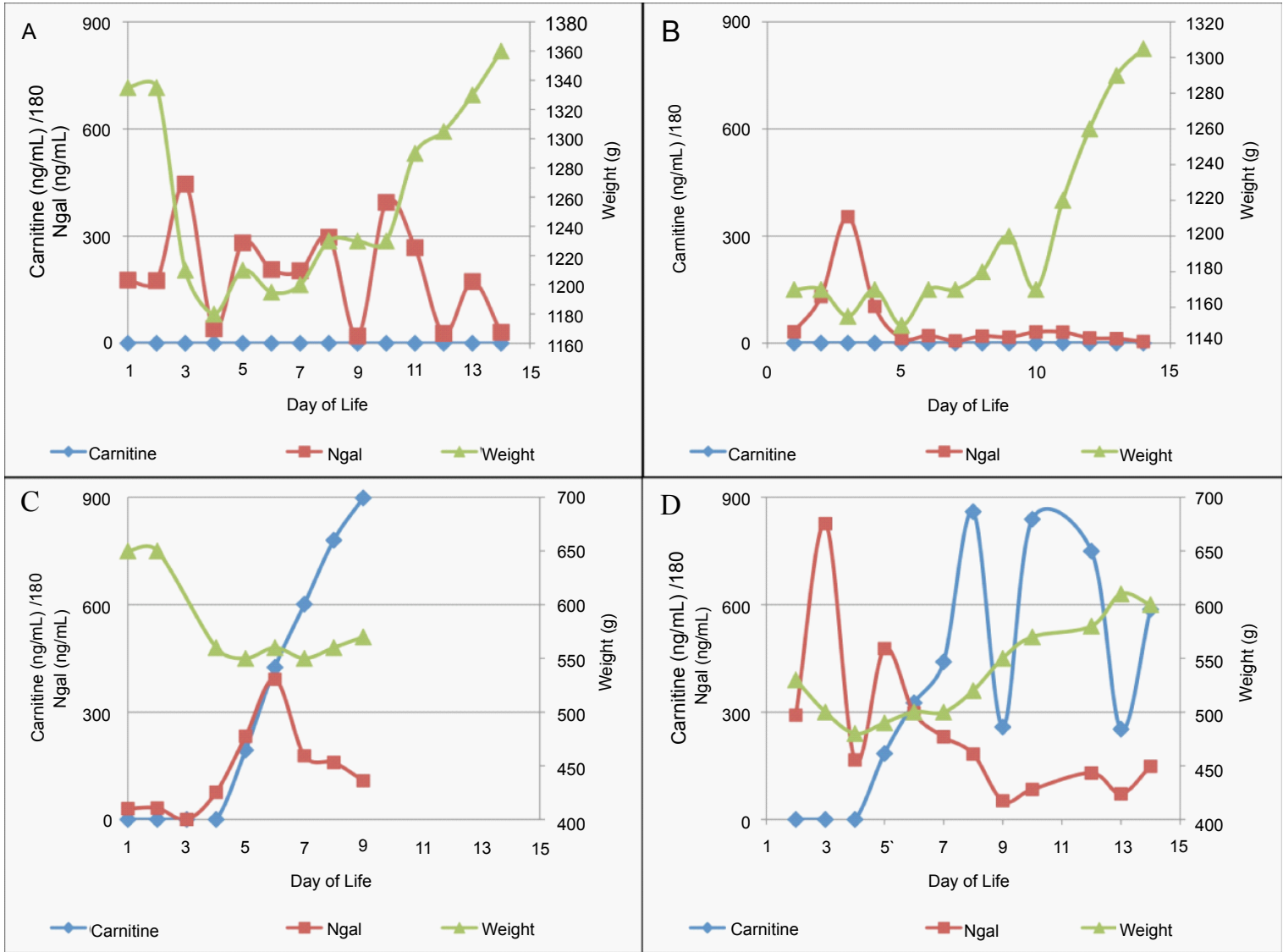

Figure 3: A-D) Graph of urinary carnitine and urinary Ngal levels, and correlated neonate weight. Figures $3 \mathrm{~A}$ and $3 \mathrm{~B}$ correspond to two carnitine-negative patients with no reported renal injury. Figures $3 \mathrm{C}$ and $3 \mathrm{D}$ correspond to two carnitine-positive patients that also had elevated urinary Ngal.

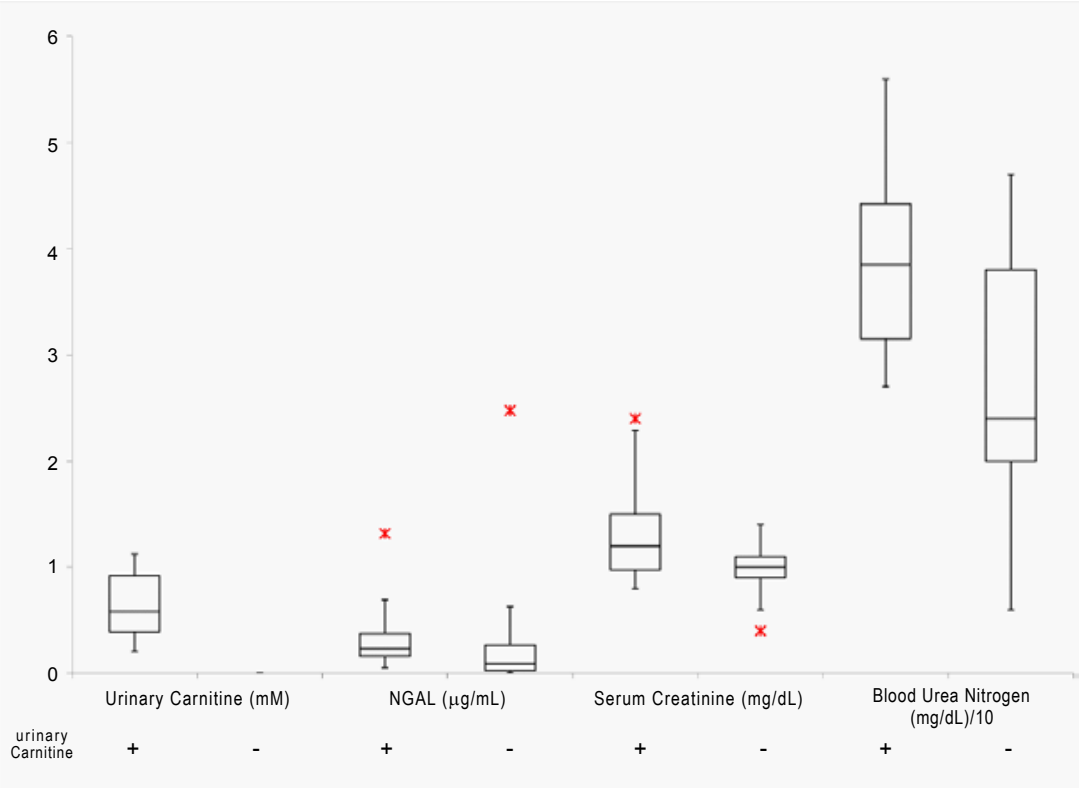

Figure 4: Box and Whisker plot comparing levels of urinary carnitine $(\mathrm{mM})$, neutrophil gelatinase-associated lipocalin $(\mathrm{NGAL})(\mu \mathrm{g} / \mathrm{mL})$, serum $\mathrm{creatinine}(\mathrm{mg} / \mathrm{dL})$, and blood urea nitrogen (BUN) (mg/dL) of carnitine-positive $(+)$ and carnitine-negative $(-)$ preterm infants. 
10 , and who presented the highest carnitine levels, received the normal carnitine dose of $20 \mathrm{mg} / \mathrm{kg} /$ day. Carnitine levels fluctuated less than urinary Ngal in these three newborns. Under normal physiological conditions, $\sim 95 \%$ of filtered carnitine is reabsorbed [32] and the kidneys play a fundamental role in maintaining carnitine homeostasis [33]. Metabolic equilibrium of carnitine is actively sustained by rate of biosynthesis [34], efficient reabsorption [35], and initial absorption of carnitine from dietary intake [36]. We hypothesize that urinary carnitine was increased in ELBW preterm infants due to failed or reduced proximal tubular reabsorption, a common symptom of ATN, which is known to be the final step leading to onset of AKI. Several contributing factors could explain increased urinary carnitine in carnitine-positive patients.

First, increased urinary carnitine could result from leakage from the kidneys into urine due to kidney damage associated with antibiotic treatment; specifically administration of gentamicin could have caused tubular damage and initiated AKI. Several antibiotics, predominantly aminoglycosides like gentamicin, cause renal tubular injury often characterized by tubular functional defects $[37,38]$. Indeed, gentamicin is a common cause of AKI [39] and administration of gentamicin could contribute to elevated urinary carnitine due to gentamicin-induced renal injury making the renal tubules incapable of reabsorbing carnitine after filtration by the glomeruli. Without normal reabsorption into the kidneys, carnitine would be expelled as urinary waste. Gentamicinassociated carnitine loss would not only compromise the renal tubules, but might also alter normal gut function and morphology given that fatty acid oxidation in epithelial cells of the intestine and colon is facilitated by carnitine [40].

Second, increased urinary carnitine could result from a primary carnitine uptake deficiency caused either by a genetic defect in, or antibiotic-associated blockage of, human organic cation transporter (hOCTN2). hOCTN2 is a sodium-ion-dependent carnitine transporter [32] known to actively transport carnitine across membranes in renal tubules [41]. A defect in, or disruption of, hOCTN2 would result in increased urinary carnitine wasting due to defective reabsorption. Many $\beta$-lactam antibiotics, such as ampicillin, block normal transport/uptake of carnitine by competitively binding to hOCTN2 in the kidneys [42]. Because the preterm infants were administered a cocktail of antibiotics including both gentamicin and ampicillin, ampicillin binding to hOCTN2 in kidney epithelial cells could block normal carnitine uptake and transport across cell membranes, leading to increased urinary carnitine. Administration of $\beta$-lactam antibiotics could increase risk of disruption of normal carnitine uptake in kidneys and cause a general increase in urinary carnitine of affected patients.

Finally, increased urinary carnitine could have been associated with indomethacin treatment. Indomethacin, a cyclooxygenase (COX) inhibitor, is commonly administered to treat patent ductus arteriosus [43].Inhibition of COX by indomethacin prevents normal decline in pulmonary vascular resistance linked to rhythmic lung distention at birth [43]. However, indomethacin also causes reduced renal blood flow [44] and almost always leads to renal failure [15]. COX inhibitors not only impair renal function by affecting renal perfusion, but also alter overall kidney development related to changes in local prostaglandin synthesis. Indomethacin has also been associated with isolated bowel perforation caused by vasoactive effects that lead to diminished mesenteric perfusion [45].

The three carnitine-positive patients were all treated either with an antibiotic cocktail containing ampicillin and gentamicin, indomethacin, or both. The carnitine-positive patient who died on DOL 10 received antibiotics for more than seven days and also received indomethacin. This patient appeared to suffer from AKI, based on elevated Ngal and carnitine levels, as well as isolated bowel perforation, which was ultimately the cause of death. We hypothesize that extended combined treatment with antibiotics and indomethacin led not only to bowel ischemia but also to AKI and renal dysfunction leading to the patient's death. The two remaining carnitine-positive ELBW patients received antibiotics or indomethacin, but not both, which could explain fluctuations in urinary carnitine and Ngal levels as well as survival through DOL [14]. Given that all twenty infants received continuous daily therapeutic carnitine, it appears that urinary carnitine in preterm infants might be used analogously to a glucose tolerance test for diabetes, i.e. healthy preterm infants appear capable of completely absorbing therapeutic doses of carnitine, whereas infants with suspected AKI cannot. Additional studies of larger preterm infant cohorts are needed to further validate carnitine in AKI diagnosis as well as effects of antibiotics and indomethacin administration on survival rates of premature neonates.

\section{Acknowledgements}

We thank the Neonatal Network Research Coordinators and Dr. Adrian Lavery for their assistance in screening and enrolling research subjects and the nursing staffs at Good Samaritan Hospital, University Hospital, and Cincinnat Children's Hospital for assistance in the specimen procurement. MAK would like to acknowledge support of Miami University and the Ohio Board of Regents for funding to establish the Ohio Eminent Scholar Laboratory where the metabonomics work was performed. Dr. Schibler and the Neonatal Network Research Coordinators are supported by a grant from NIH/NICHD (U10-HD027853). Dr. Kennedy is supported by a grant from the $\mathrm{NIH} / \mathrm{NCl}(1 \mathrm{R} 15 \mathrm{CA} 152985)$.

\section{References}

1. Andreoli SP (2009) Clinical Evaluation of Acute Kidney Injury in Children Pediatr Nephrol 10: 1603-1618.

2. Williams DM, Sreedhar SS, Mickell JJ, Chan JCM (2002) Acute Kidney Failure Arch Pediatr Adolesc Med 156: 893-900.

3. Mehta R, Kellum J, Shah S, Molitoris B, Ronco C, et al. (2007) Acute Kidney Injury Network: report of an initiative to improve outcomes in acute kidney injury. Critical Care 11: R31.

4. Zappitelli M, Goldstein SL (2009) Acute Kidney Injury: General Aspects Pediatric Nephrology in the ICU, eds Kiessling SG, Goebel J, \& Somers MJG (Springer, Berlin Heidelberg) 85-97.

5. Gill N, Nally JV, Fatica RA (2005) Renal Failure Secondary to Acute Tubula Necrosis. Chest 128: 2847-2863.

6. Coca SG, Parikh CR (2008) Urinary Biomarkers for Acute Kidney Injury: Perspectives on Translation. Clin J Am Soc Nephrol 3: 481-490.

7. Vaidya VS, Waikar SS, Ferguson MA, Collings FB, Sunderland K, et al. (2008) Urinary Biomarkers for Sensitive and Specific Detection of Acute Kidney Injury in Humans. Clin Transl Sci 1: 200-208.

8. Parikh CR, Jani A, Melnikov VY, Faubel S, Edelstein CL (2004) Urinary interleukin-18 is a marker of human acute tubular necrosis. Am J Kidney Dis 43: $405-414$

9. Lavery AP, Meinzen-Derr JK, Anderson E, Ma Q, Bennett M, et al. (2008) Urinary NGAL in premature infants. Pediatr Res 64: 423-428.

10. Hans W, Walkar SS, Johnson A, Betensky R, Dent C, et al. (2008) Urinary biomarkers in the early diagnosis of acute kidney injury MMP-9, NAG, and KIM1 in acute kidney injury. Kidney Int 73: 863-869.

11. Haase M, Bellomo R, Devarajan P, Schlattmann P, Haase-Fielitz A (2009) Accuracy of neutrophil gelatinase-associated lipocalin (NGAL) in diagnosis and prognosis in acute kidney injury: A systematic review and meta-analysis. Am J Kidney Dis 54: 1012-1024.

12. Askenazi DJ, Griffin R, McGwin G, Carlo W, Ambalavanan N (2009) Acute kidney injury is independently associated with mortality in very low birthweight infants: a matched case-control analysis. Pediatr Nephrol 24: 991-997.

13. Tugay S, Bircan Z, Çağlayan Ç, Arısoy A, Gökalp A (2006) Acute effects of 
Citation: Romick-Rosendale LE, Schibler KR, Kennedy MA (2012) A Potential Biomarker for Acute Kidney Injury in Preterm Infants from Metabolic Profiling. J Mol Biomark Diagn S3:001. doi:10.4172/2155-9929.S3-001

gentamicin on glomerular and tubular functions in preterm neonates. Pediatr Nephrol 21: 1389-1392.

14. Lew SQ, Borum ML, Ing TS (2010) Gastrointestinal Complications of Acute Kidney Injury. Management of Acute Kidney Problems 4: 209-220

15. Betkerur MV, Yeh TF, Miller K, Glasser RJ, Pildes RS (1981) Indomethacin and Its Effect on Renal Function and Urinary Kallikrein Excretion in Premature Infants with Patent Ductus Arteriosus 68: 99-102.

16. Zappitelli M, Goldstein SL (2010) Acute Kidney Failure in Children. Management of Acute Kidney Problems 459-467.

17. Soni SS, Cruz D, Bobek I, Chionh CY, Nalesso F, et al. (2010) NGAL: a biomarker of acute kidney injury and other systemic conditions. Int Urol Nephrol 42: 141-150.

18. Mishra J, Dent C, Tarabishi R, Mitsnefes MM, Ma Q, et al. (2005) Neutrophil gelatinase-associated lipocalin (NGAL) as a biomarker for acute renal injury after cardiac surgery. The Lancet 365: 1231-1238.

19. Goetz DH, Holmes MA, Borregaard N, Bluhm ME, Raymond KN, et al. (2002) The Neutrophil Lipocalin NGAL Is a Bacteriostatic Agent that Interferes with Siderophore-Mediated Iron Acquisition. Mol cell 10: 1033-1043.

20. Nielsen BS, Borregaard N, Bundgaard JR, Timshel S, Sehested M \& Kjeldsen $L$ (1996) Induction of NGAL synthesis in epithelial cells of human colorectal neoplasia and inflammatory bowel diseases. Gut 38: 414-420.

21. Lindon JC, Holmes E, Nicholson JK (2001) Pattern recognition methods and applications in biomedical magnetic resonance. Prog Nucl Mag Res Sp 39 $1-40$.

22. Lindon JC, Nicholson JK, Holmes E, Everett JR (2000) Metabonomics: Metabolic processes studied by NMR spectroscopy of biofluids. Concept Magnetic Res 12: 289-320.

23. Nicholson JK, Wilson ID (1989) High-resolution proton magnetic-resonance spectroscopy of biological-fluids. Prog Nucl Mag Res Sp 21: 449-501.

24. Nicholson JK, Lindon JC, Holmes E (1999) Metabonomics: understanding the metabolic responses of living systems to pathophysiological stimul via multivariate statistical analysis of biological NMR spectroscopic data. Xenobiotica 29: 1181-1189.

25. Rousseau R, Govaerts B, Verleysen M, Boulanger B (2008) Comparison of some chemometric tools for metabonomics biomarker identification. Selected papers presented at the Chemometrics Congress "CHIMIOMETRIE 2006" Paris, France, 30 November - 1 December 2006 91: 54-66.

26. Goodpaster AM, Kennedy MA (2011) Quantification and statistical significance analysis of group separation in NMR-based metabonomics studies. Chemometr Intell Lab Syst 109: 162-170

27. Abosaif NY, Tolba YA, Heap M, Russell J, Nahas AME (2005) The Outcome of Acute Renal Failure in the Intensive Care Unit According to RIFLE: Mode Application, Sensitivity, and Predictability. Am J Kidney Dis 46: 1038-1048.

28. Levey AS, Bosch JP, Lewis JB, Greene T, Rogers N et al.(1999) A More Accurate Method To Estimate Glomerular Filtration Rate from Serum Creatinine: A New Prediction Equation. Ann Intern Med 130: 461-470.
29. Perrone RD, Madias NE, Levey AS (1992) Serum creatinine as an index of renal function: new insights into old concepts. Clin Chem 38: 1933-1953.

30. Levey AS (1990) Measurement of renal function in chronic renal disease Kidney Int 38: 167-184.

31. Quaggin SE, Kreidberg JA (2008) Development of the renal glomerulus: good neighbors and good fences. Development 135: 609-620.

32. Haschke M, Vitins T, Lude S, Todesco L, Novakova K, et al. (2010) Urinary excretion of carnitine as a marker of proximal tubular damage associated with platin-based antineoplastic drugs. Nephrol Dial Transpl 25: 426-433.

33. Rebouche CJ, Seim H (1998) Carnitine metabolsim and its regultation in microorganisms and mammals. Annu Rev of Nutr 18: 39-61.

34. Carter AL, Abney TO, Lapp DF (1995) Biosynthesis and Metabolism of Carnitine. J Child Neurol 10: 2S3-2S7.

35. Engel AG, Rebouche CJ, Wilson DM, Glasgow Am, Romshe CA (1981) Primary systemic carnitine dehciency. II. Renal handling of carnitine. Neurology 31: 819.

36. Rebouche CJ, Chenard CA (1991) Metabolic Fate of Dietary Carnitine in Human Adults: Identification and Quantification of Urinary and Fecal Metabolites. J Nutr 121: 539-546.

37. Giapros VI, Andronikou S, Cholevas VI, Papadopoulou ZL (1995) Renal function in premature infants during aminoglycoside therapy. Pediatr Nephrol 9: 163-166.

38. Giapros VI, Andronikou SK, Cholevas VI, Papadopoulou ZL (2003) Rena function and effect of aminoglycoside therapy during the first ten days of life. Pediatr Nephrol 18: 46-52.

39. Selby NM, Shaw S, Woodier N, Fluck RJ, Kolhe NV (2009) Gentamicinassociated acute kidney injury. QJM 102: 873-880.

40. Shekhawat PS, Srinivas SR, Matern D, Bennett MJ, Boriack R, et al. (2007) Spontaneous development of intestinal and colonic atrophy and inflammation in the carnitine-deficient jvs (OCTN2-/-) mice. Mol Genet Metab 92: 315-324.

41. Ohashi R, Tamai I, Yabuuchi H, Nezu JI, Oku A, et al. (1999) Na+ Dependent Carnitine Transport by Organic Cation Transporter (OCTN2): Its Pharmacological and Toxicological Relevance. J Pharmacol Exp Ther 291 778-784.

42. Ganapathy ME, Huang W, Rajan Dp, Carter AL, Sugawara M, et al. (2000) $\beta$-Lactam Antibiotics as Substrates for OCTN2, an Organic Cation/Carnitine Transporter. J Biol Chem 275: 1699-1707.

43. Gournay V (2005) Complications of Cyclooxygenase Inhibition in Preterm Infants. Interventions for Persisting Ductus Arteriosus in the Preterm Infant 74 83.

44. Pezzati M, Vangi V, Biagiotti R, Bertini G, Cianciulli D, et al. (1999) Effects of indomethacin and ibuprofen on mesenteric and renal blood flow in preterm infants with patent ductus arteriosus. J Pediatr 135: 733-738.

45. Shorter NA, Liu JY, Mooney DP, Harmon BJ (1999) Indomethacin-associated bowel perforations: A study of possible risk factors. J Pediatr Surg 34: 442-444. 